\title{
Sizes of quiet Sun transition region structures
}

\author{
C. Gontikakis ${ }^{1}$, H. Peter ${ }^{2}$, and H. C. Dara ${ }^{1}$ \\ 1 Research Center for Astronomy and Applied Mathematics, Academy of Athens, Greece \\ e-mail: edara@cc.uoa.gr \\ 2 Kiepenheuer-Institut für Sonnenphysik, Schöneckstr. 6, 79104 Freiburg, Germany \\ e-mail: peter@kis.uni-freiburg.de
}

Received 26 September 2002 / Accepted 16 June 2003

\begin{abstract}
We studied the morphology of the transition region of the quiet Sun, with data obtained by the Solar Ultraviolet Measurements of Emitted Radiation spectrometer (SUMER) and the Extreme-Ultraviolet Imaging Telescope (EIT), in September 1996. We analyzed lines emitted in the chromosphere, the low transition region and the low corona. We computed the mean autocorrelation function for the radiance images in order to estimate the characteristic size of the features present in the transition region. Moreover, we calculated autocorrelation functions for the dopplergrams and line width images deduced from the SUMER data. In addition to the line core of the C IV line, we investigated a broader tail component, whose origin is still unclear. We found that the size of the bright radiance features is always larger than the size of the structures of the dopplergrams and Doppler widths, at any altitude. The network features seem to diminish at a temperature around $10^{5} \mathrm{~K}$, due to the thermodynamic properties of the transition region. The mean size of the structures of the tail component radiance is smaller than the one of the core radiance.
\end{abstract}

Key words. Sun: chromosphere - Sun: corona - Sun: transition region

\section{Introduction}

Lately, there is a general consensus that the transition region (TR) between the chromosphere and the corona, plays an important rôle for the acceleration of the fast solar wind (Hansteen \& Leer 1995; Hassler et al. 1999) and is involved in the coronal heating phenomenon (Hansteen 1993; Chae et al. 1998; Gontikakis \& Dara 2000). The systematic study of this part of the solar atmosphere, which emits mostly in the EUV, started after the first space missions that made observations of the Sun in this spectral domain possible. Such missions were the OSO (Orbiting Solar Observatory) satellites, Skylab, SMM (Solar Maximum Mission), HRTS (High Resolution Telescope and Spectrograph) and, recently, SOHO (Solar and Heliospheric Observatory, Domingo et al. 1995) and TRACE (Transition Region and Coronal Explorer, Handy et al. 1999).

A first result about the quiet Sun transition region is that the chromospheric network is still a characteristic feature of its morphology (Reeves 1976). Gabriel (1976), using a MHD calculation, showed that the magnetic field, confined at the boundaries of the supergranulation cells at the level of the chromosphere due to the motion of the convection zone, expands with height covering all the space at the coronal level. These expanding magnetic features, called funnels, are believed to be the sites of the solar wind acceleration or, perhaps, the

Send offprint requests to: C. Gontikakis, e-mail: cgontik@cc.uoa.gr footpoints of quiet Sun loops connecting network features of different supergranulation cells. The variation of the width of the network boundaries with height, could be an indication of the expansion of the funnel-type magnetic field (Patsourakos et al. 1999).

The intensities of lines from the low corona were interpreted by Gabriel (1976), who assumed that the funnels were heated by conduction from the corona. However, the brightness of the low $\left(\simeq 10^{5} \mathrm{~K}\right)$ transition region computed for his model by using the Differential Emission Measure (DEM) curve was lower than observed. To explain this discrepancy, Dowdy et al. (1986) proposed that the energy responsible for the large brightness of the low transition region is produced in small loops, confined within the network boundaries. Feldman (1983, 1987) and Feldman et al. (2001) suggested that the TR plasma, from $2 \times 10^{4} \mathrm{~K}$ to $7 \times 10^{5} \mathrm{~K}$, seems to be confined in small loop-like features, which do not extend to the corona, nor do they have a chromospheric counterpart. This picture is different from the standard view that the TR acts as an interface between the chromosphere and the corona. However, the question on the connection of the chromosphere and the transition region remains open. Judge \& McIntosh (1999) compared different views of the transition region and argued that even if the chromosphere and the transition region look disconnected, they might well be connected. This could be an effect of the intrinsically dynamic nature of the transition region (Wikstøl et al. 1998). 
The shape and Doppler shift of emission lines inform us about two properties of the transition region: first, that the lines emitted from a substantial part of the solar surface are, on the average, redshifted (Doscheck et al. 1976) and, second, that the shape of the line profiles presents deviations from a single Gaussian profile. Detailed studies of the redshift effect show that it is more pronounced at temperatures of $\simeq 10^{5} \mathrm{~K}$, whereas it is decreasing and turns to blueshift, for lines emitted in the low corona (Peter \& Judge 1999; Teriaca et al. 1999). A statistical analysis shows that the redshift tends to be stronger for brighter individual profiles (Brynildsen et al. 1998). As for the profile, Kjeldseth Moe \& Nicolas (1977) were the first to notice a second Gaussian component in the enhanced wings of the transition region spectral lines.

The SUMER (Wilhelm et al. 1995) spectrograph, with its high spectral and spatial resolutions, has given the opportunity to systematically study the two spectral components in the lines of the transition region, mostly in the line of C IV (Peter 2000, 2001). The picture deduced from these studies is that the two spectral components correspond to different physical regimes. This is so, because the two components show different correlations between the different spectral parameters (radiance, Doppler shift and line width). It seems that the core components are a trace of the small loops, whereas the tail components are emitted by the funnels.

In this work we carried out a quantitative study of the morphology of these spectral components. To do that, we computed the mean autocorrelation function (ACF) of the three types of images that could be deduced from the spectral and spatial information taken from SUMER, i.e. radiance images, dopplergrams and maps of the spectral line widths. In the same way we analyzed EIT images that where obtained during the SUMER raster. The feature size determination is performed again on the images of the tail component of the $\mathrm{C}$ IV line, but there the low signals lead to less conclusive results.

\section{Observations and data reduction}

\subsection{Observations}

Part of the data used for the present study were obtained by a SUMER raster scan on 22 September 1996, during solar minimum (see Fig. 1). They covered a quiet Sun region of $540^{\prime \prime} \times$ $300^{\prime \prime}$, at $25^{\circ}$ heliocentric angle to the north-east. The step size was $3^{\prime \prime}$ for the 181 horizontal steps of the scan, with a slit width of $1^{\prime \prime}$. The spectral region ( $1531 \AA$ to $1552 \AA$ ) includes, among others, the C IV $1548 \AA$ line, the Si II $1533 \AA$ one and the Ne VIII $770 \AA$ one, in second order. The SUMER data were binned along the slit on the ground and this, along with the step process (under-sampling) leads to an effective size of the resolution element of $3^{\prime \prime} \times 3^{\prime \prime}$. The exposure time at each slit position was $150 \mathrm{~s}$ so that the duration of the scan was of $7 \mathrm{~h}$ and $50 \mathrm{~min}$, starting at 00:40 UTC.

The corresponding EIT images were selected within the period of the SUMER observational time in the He II $304 \AA$, the Fe IX/X $171 \AA$, the Fe XII/XXIV $195 \AA$, and the Fe XV $284 \AA$ lines. They provided synoptic, $1024 \times 1024$ pixels, full disk images in the four pass bands, with a pixel size of $2.6^{\prime \prime} \times 2.6^{\prime \prime}$ and full disk images with reduced spatial resolution. The exposure time used varied for different images from approximately $3 \mathrm{~s}$ to 2 min, depending on the line and the spatial pixel binning. The high-resolution EIT images were taken during the first hour of the SUMER scan, whereas most of the low-resolution ones, during the seventh hour. EIT images are snapshots of the studied area and are meant to be used in a complementary way in this analysis. Let us note that the same SUMER data set was used by Hassler et al. (1999) to study the solar wind, by Dammasch et al. (1999) to measure the reference wavelength of Ne VIII, and by Peter (2000) to study the tail component of C IV.

\subsection{Data reduction}

For the EIT images, we followed the standard data treatment proposed by the instrument team, including: background subtraction, degridding, flat field correction and degradation correction. Similarly, for the SUMER data, flat field and geometrical distortions, as well as additional corrections were applied (see Peter 1999 for more details).

The Gaussian fits to the spectral profiles and especially the decomposition of the core and tail components in the case of the C IV line follows exactly the procedure described in detail by Peter (2000). A genetic algorithm based, optimization method was used (Charbonneau 1995).

One of the goals of this work is the study of the quiet Sun morphology and the evolution of its features with height. The ACF based on Fourier analysis, provides a statistical estimation of the scale length of the bright features of an image. The smaller the features, the sharper the central peak of the ACF will be. We computed the map of the ACF in 2-D and, using polar coordinates, we calculated the mean ACF along the radial coordinate $r$, after averaging over $\phi$. Before the calculation of the ACF, we applied a cosine bell function in order to smooth the discontinuity at the image borders, thus we overcame the limited size of our sample (Brault \& White 1971).

\section{Data analysis}

\subsection{Artificial data}

In order to make clear the results of the autocorrelation operation, we first applied it to artificially created radiance images. The images contained several circular features, either randomly distributed or ordered in a chessboard and in a hexagonal arrangement (see Fig. 2). We checked if the Half Width at Half Maximum (HWHM) of the central peak of the ACF is close enough to the feature size. The "radiance" of the features is either uniform or Gaussian with the peak at the center of the features. The tests performed show that the best fit between the ACF $(H W H M)$ and the size of the features is obtained for Gaussian radiance distribution features. In this case the radius is equal to the width $(1 / e)$ of the distribution. The ACF contains also a secondary peak that corresponds to the mean distance between the features. The amplitude of the second peak is much smaller than the central one, due to the average over azimuth angle $\phi$. 


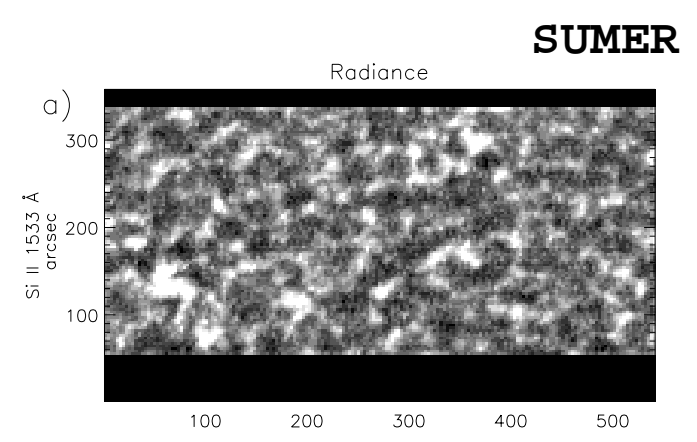

\section{images}
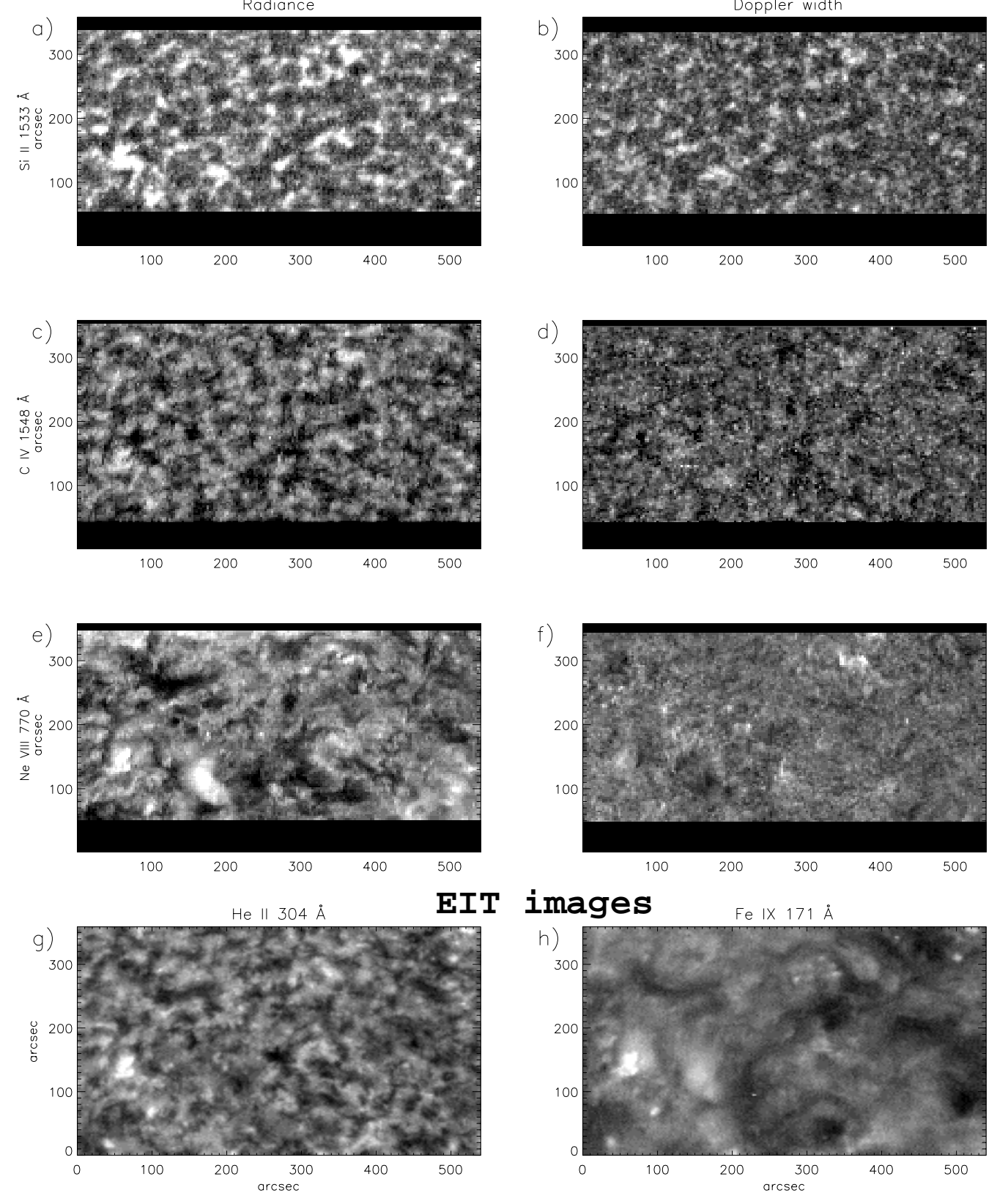

Fig. 1. The SUMER images (first three rows) for the a), b) Si II, c), d) C IV, and e), f) Ne VIII lines. The left column shows the radiances and the right the Doppler widths. The last row g), h) shows the EIT image of the He II $304 \AA(01: 30: 13$ UTC) and of Fe IX/X 171 $\AA$ line (01:00:14 UTC). The full-disk EIT images were cut and co-aligned to match the SUMER FOV.

In order to deal with dopplergrams, where we have redshifted (positive velocities) and blueshifted (negative velocities) features, we created an image with positive and negative features (as mountains and ravines). There, the HWHM of the ACF is between the sizes of the two types of features. If we change the sign of the features, the $H W H M$ of the ACF has a relative variation of only $0.5 \%$; the autocorrelation operation does not distinguish between positive and negative features. Thus, the HWHM of the ACF of a dopplergram takes into account both directions of flow.
For different radiance distributions of the circular features the relation between the $H W H M$ and the feature size is $H W H M \simeq 0.8 \rho$, where $\rho$ is the structure radius, for sizes smaller than 10 pixels. However, the relation is not linear when the size is larger (Fig. 3). Our tests with square features have shown that this result is independent of the shape of the features and our method reproduces quite well the sizes of different shapes, provided they are not very large. 

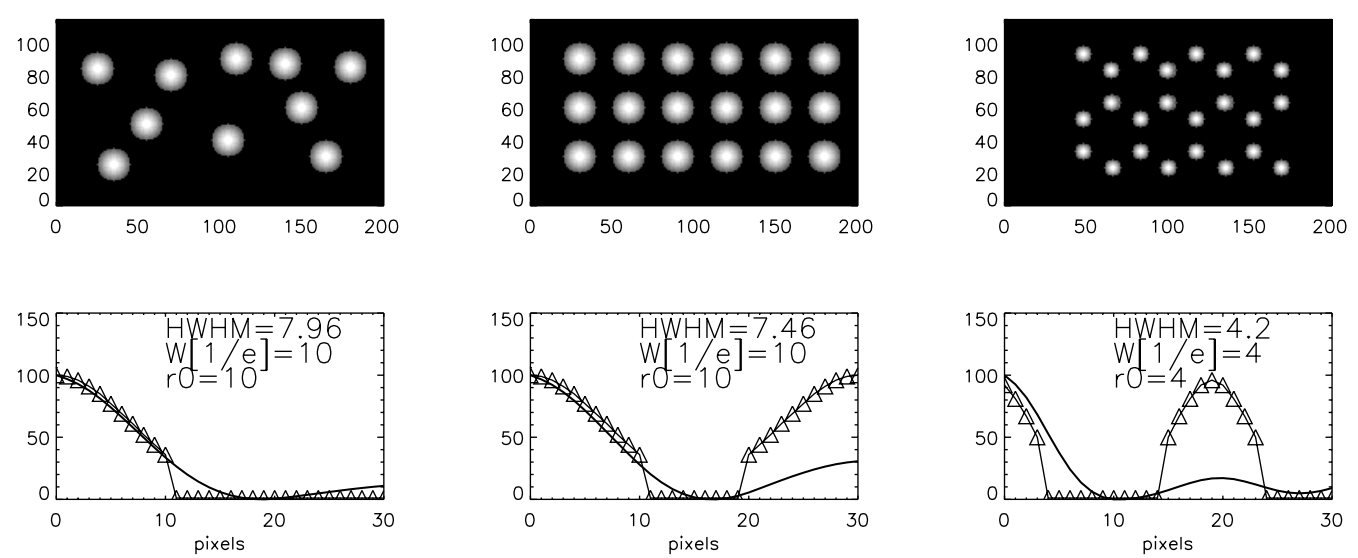

Fig. 2. Top row: three examples of artificial radiance images with circular features: randomly distributed, in a chessboard pattern, or in a network of hexagons. Each feature is circular with radius $r_{0}$, with a Gaussian radiance distribution of width $W[1 / e]$ equal to the radius. The lower row shows the radiance variation along the radius of a typical feature (triangles) and the mean ACF (thick solid line). The first peak of the ACF fits the typical features, whereas the second, smaller peak, is at a distance equal to the mean distance between the closest features.

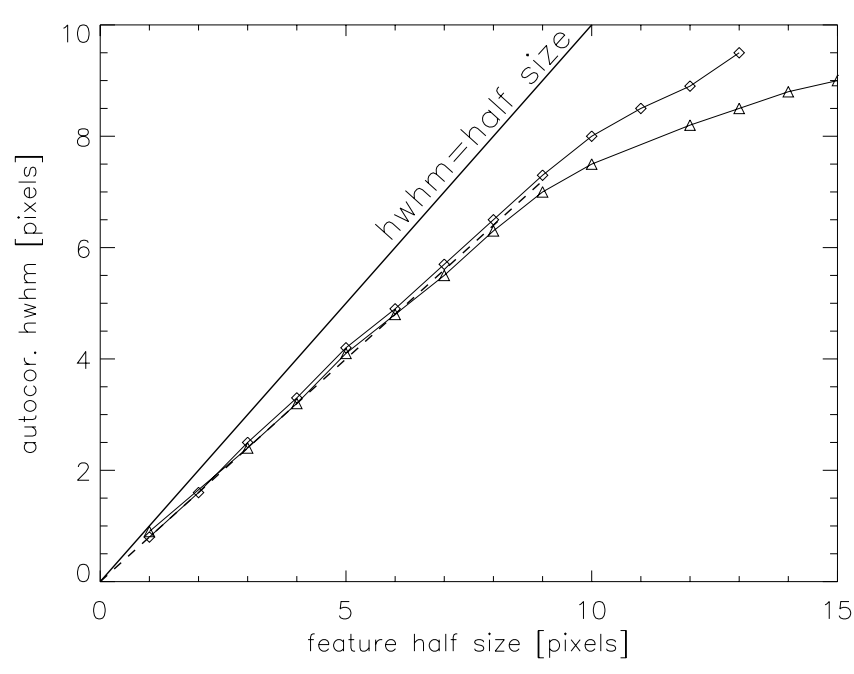

Fig. 3. Dependence of the $H W H M$ on the size of circular features for different radiance distributions. The features with uniform radiance distribution are represented by triangles and the ones with Gaussian radiance distribution by diamonds. The line corresponding to $H W H M$ equal to the feature width is also traced. The dashed line, correspond to $0.8 \times H W H M$.

\subsection{Influence of noise}

To estimate the influence of the noise on the autocorrelation images, we chose the radiance image of Si II $1533 \AA$, where the noise is small. The size of the pixel corresponds to approximately $3^{\prime \prime} \times 3^{\prime \prime}$ (see Sect. 2.2). We progressively added noise to this image, in order to follow the change of the ACF shape. When we add e.g. $15 \%$ noise in the Si II image, this means that at each pixel $(i, j)$ of the initial image, of radiance $I(i, j)$ we add a random number within the range $\pm 0.15 I(i, j)$. In Fig. 4 , we compare the ACF of Si II with the one of Si II plus a contribution of $15 \%$ and $50 \%$ relative noise and the ACF of an image having just noise. The addition of noise reduces the $H W H M$ of the ACF. The ACF of an image with noise only (which means just filled with random numbers) will have a $H W H M$ near to half the pixel size (3"/2 for the example of Fig. 4).

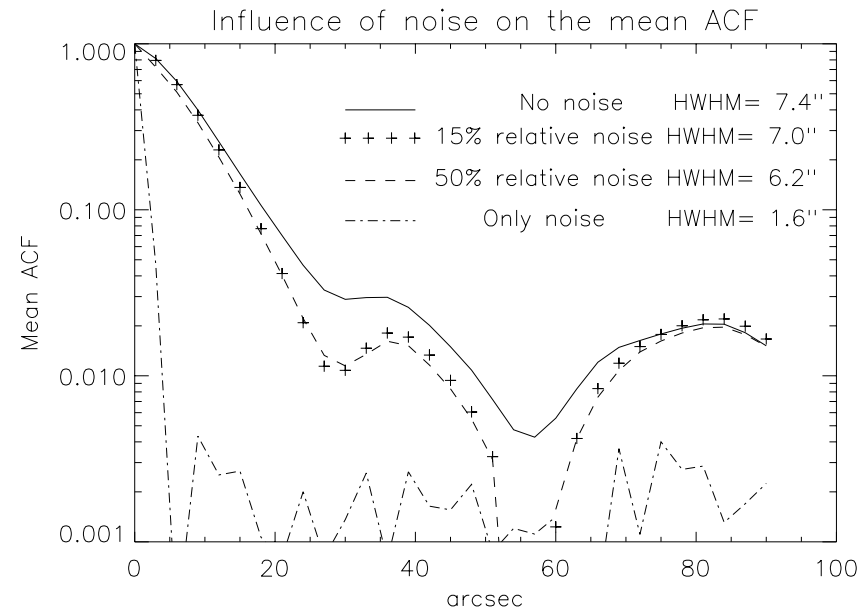

Fig. 4. Autocorrelation curves of images affected by noise. The solid line corresponds to the Si II 1533 A image without noise addition, the crosses to $15 \%$ relative noise addition, the dashed line to $50 \%$ relative noise addition and the point-dashed one to a fully noisy image. For this last case, the $H W H M$ of the ACF is equal to half the pixel size $\left(1\right.$ pixel $\left.=3^{\prime \prime} \times 3^{\prime \prime}\right)$.

For the radiance images, we estimated the percentage of the noise and how it could influence the resulting ACF. We used artificial images, as the one in the first panel of Fig. 2, where an amount of noise, corresponding to the counting statistics of the image, is taken into account. Since we knew in advance the size of the artificial features, we could estimate the new $H W H M$ after the noise addition. Thus we could find the dependence of $H W H M$ on noise.

We concluded that for the radiance images of the Si II, C IV and $\mathrm{Ne}$ VIII spectral lines the relative noise is less than $10 \%$ and does not influence significantly the resulting ACF. For the continuum, the He II image and the radiance of the tail component of C IV the relative noise is of $15 \%$ and we estimated that the relative uncertainty when measuring the $H W H M$ is about $4 \%$. These noise estimations from the artificial images are in agreement with those presented in Fig. 4 resulting from a solar image. 

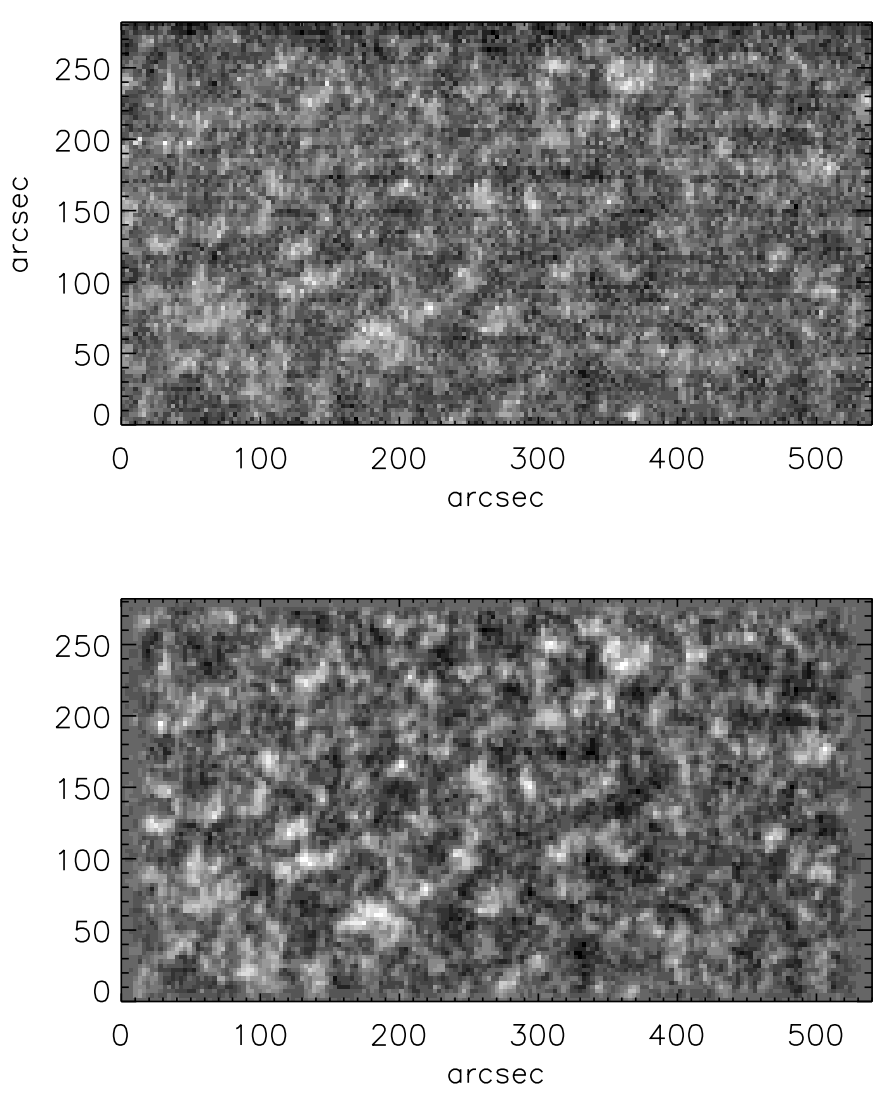

Fig. 5. The Si II $1535 \AA$ A non-thermal width map before (top) and after (bottom) the appropriate treatment (see Sect. 3.2). The treated image is less spiky due to the smoothing we applied to smear out the noise. The horizontal stripes present in the upper image, due to imperfections of the flat field, are also corrected (see Dammasch et al. 1999).

In the case of the dopplergrams and the non-thermal width maps, the noise is more important as it is clear from the images themselves (see in Fig. 1 the non-thermal width maps). In order to reduce the effect of the noise, we smoothed these images by convolving them with a sharp 2D-Gaussian having a width of $6^{\prime \prime}$ for the C IV dopplergram, 7.5" for the continuum image, $9^{\prime \prime}$ for the non-thermal width maps of Si II, C IV and $\mathrm{Ne}$ VIII, as well as for the Ne VIII dopplergram, while $12^{\prime \prime}$ for the Si II dopplergram. The width of the Gaussian was chosen in such a way, so we could reduce the noise with a minimum smearing of the features.

In Fig. 5 we show the non-thermal width map of the Si II $1533 \AA$, line before and after the smoothing. With the exception of the continuum image, we didn't apply such a treatment to the radiance images since their noise level is very low. It is worth noting that when the smoothing of two pixels (i.e. 6") is applied, the network shows up clearly even in the line width map (cf. Fig. 5).

\section{Typical size of quiet Sun structures}

In Fig. 6, we show the mean ACF for the maps of the radiance, line shift and non-thermal width for Si II, the C IV core component (CC) and Ne VIII, along with the continuum and the EIT He II $304 \AA$ image. We computed the non-thermal width, $\xi$, considering that the $1 / e$ line width, $w$, is expressed
Table 1. Formation temperatures of the spectral lines, the $H W H M$ of the features of the radiance images and an estimation of its uncertainty.

\begin{tabular}{lllll}
\hline \hline & $\lambda(\AA)$ & $\log T(\mathrm{~K})$ & $H W H M\left({ }^{\prime \prime}\right)$ & $\Delta H W H M\left({ }^{\prime \prime}\right)$ \\
& & & & \\
\hline Si I cont. & 1533 & 3.7 & $4.0-5.2$ & $\ldots$ \\
Si II & 1533 & 4.4 & 7.4 & 0.1 \\
He II & 304 & 4.7 & 9.2 & 0.3 \\
C IV & 1548 & 5.0 & 5.5 & 0.1 \\
Ne VIII & 770 & 5.8 & 11 & 0.05 \\
\hline
\end{tabular}

by the ion sound velocity, $v_{\mathrm{s}}$, and the non-thermal velocity, $\xi$, as: $w=\sqrt{2 v_{\mathrm{s}}^{2}+\xi^{2}}$ (Mariska 1992).

In the corresponding panels of Fig. 6, we present the $H W H M$ of the ACF with and without the smoothing of the image. The first one corresponds to the lower limit, with the given resolution of $3^{\prime \prime}\left(H W H M=1.5^{\prime \prime}\right)$, while the second one corresponds to the upper limit, given that in the smoothed image the feature size may be overestimated. The Si I continuum panel corresponds to a height below the canopy of the magnetic field, whereas, the other images to a height above it.

The ACF graphs show a first peak at $r=0^{\prime \prime}$ and, in most of the cases, a second one at $r \cong 30-40^{\prime \prime}$. The parameter we are interested in is the $H W H M$ of the first peak of the ACF functions, which represents the size of the features, as already mentioned in Sect. 3.1. The presence of the second peak may correspond to the mean distance between the features which, in our case, is the size of the supergranulation network, its low amplitude being due to the average over $\phi$. Other peaks, for $r$ larger than $40^{\prime \prime}$, are probably due to the periodic nature of the supergranulation network.

For the ACF calculated for the radiance images, we can conclude that the HWHM is increasing with the formation temperature of the lines, with the exception of C IV. This result seems to be significant since the difference of these values are larger than the estimated error bars. The $H W H M$ of the nonthermal width images shows a variation with temperature, similar to the one of the radiance images. Comparing the ACF $H W H M$ of the same line in different images (radiance, dopplergram, and non-thermal width), we found that the HWHM of the radiance images is larger than that of the dopplergrams and of the non-thermal width maps, even for the smoothed ones. To compare the variation of the structure sizes determined from the ACFs, we plot the HWHM for the various ACFs as a function of formation temperature in Fig. 7 (we omitted the value for He II as it was recorded with EIT, while the rest ACFs are based on SUMER data). Even though the values for the $H W H M$ of the ACFs for Doppler shift and non-thermal width are close to the resolution limit, they closely follow the trend of the structure sizes seen in the radiance images.

Table 1, contains the formation temperature of the lines and continuum, the $H W H M$ of the features of the radiance images and an estimation of its uncertainties. The formation temperatures of Si II, C IV, and Ne VIII are taken from Chae et al. (1998). They represent the maximum of the contribution function of the line, $\left(n_{\mathrm{i}} / n_{\mathrm{el}}\right) T^{-1 / 2} \exp [-E /(k T)]$, where $\left(n_{\mathrm{i}} / n_{\mathrm{el}}\right)$ is the ionisation fraction and $E=h c / \lambda$ is the energy of the 


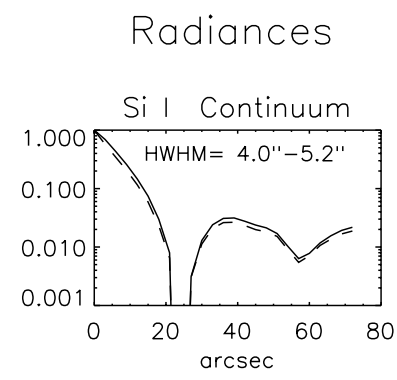

\section{Doppler shifts Non-thermal widths $\xi$}
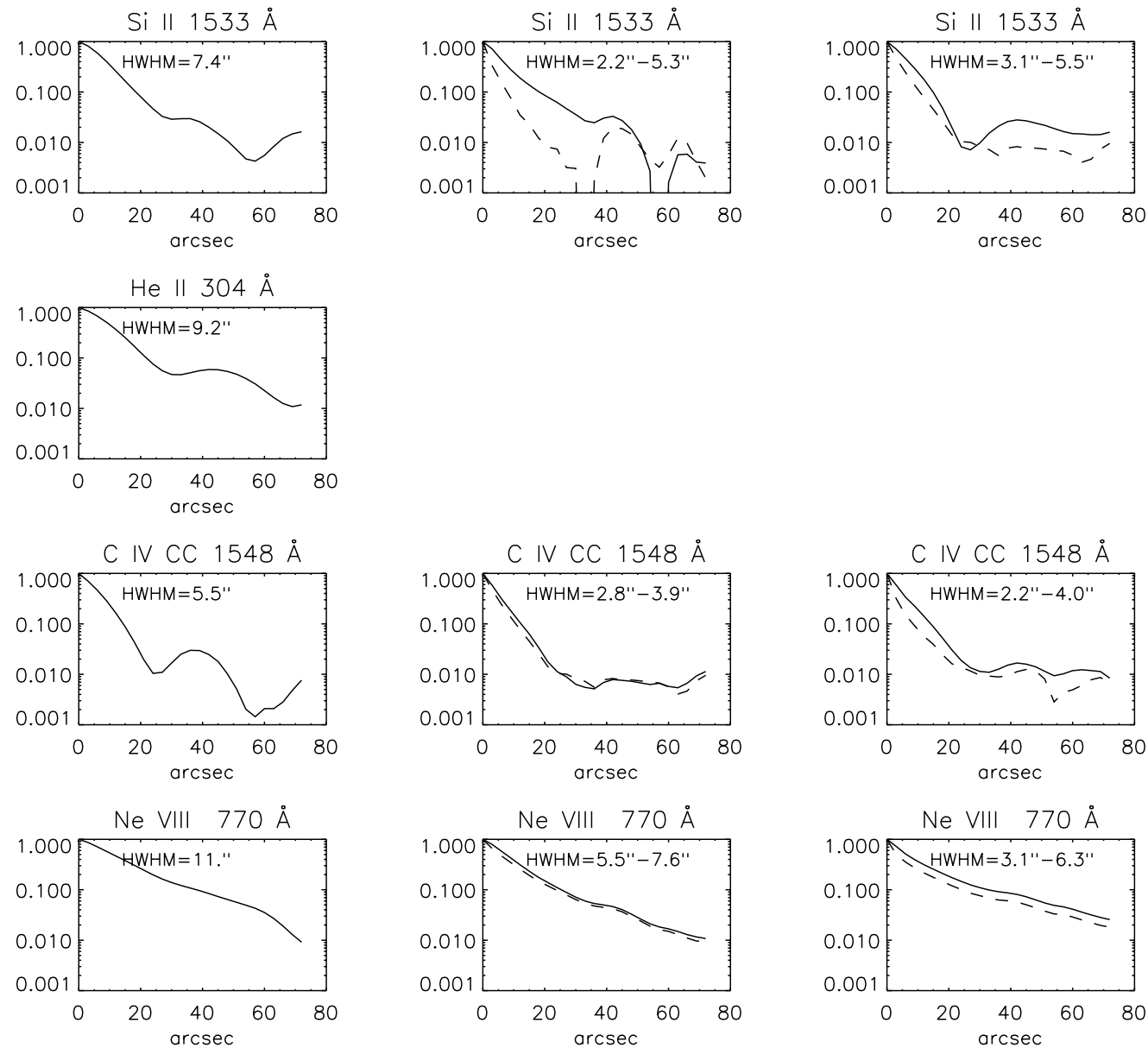

Fig. 6. ACF of all images except those of the $\mathrm{C}$ IV tail component (TC) and the three EIT coronal lines. In the first column, we show the ACF for the radiance images: the continuum near $1533 \AA$, the Si II $1533 \AA$, the core component (CC) of the C IV $1548 \AA$, the He II, being the only EIT image used, and of the Ne VIII $770 \AA$ A. The second column shows the ACF for the dopplergrams for the SUMER data, with the same order (Si II, C IV, Ne VIII). The third column shows the ACF of non-thermal width images, smoothed (solid line) and unsmoothed (dashed line). In each panel we note the $H W H M$ of the ACF, the second one corresponding to smoothed images.

transition. In the case of He II, we only list the temperature of maximum ion fraction following Arnaud \& Rothenflug (1985). Please note that this can only be a rough indicator, as the formation of the helium lines is still poorly understood (see e.g. Andretta et al. 2003). For the formation of the Si I, continuum near $1530 \AA$, we adopted the temperature at the maximum of the contribution function in the semi-empirical model of Vernazza et al. (1981)

The second $H W H M$ value of the Si I continuum, is calculated after the smoothing of the image (see Sect. 3.2).

The peak at $40^{\prime \prime}$, is visible in all the radiance images except the one of Ne VIII, which certifies the appearance of the supergranulation network pattern in the upper chromosphere and the low transition region up to the Ne VIII formation level. The supergranulation network is also present in the non-thermal width $\mathrm{ACF}$ of the Si II line, as well as, with a smaller contrast, in the one of C IV line. The smaller contrast maybe due to the removal of the spectral tail component, which enhances the line width at the sites of the network boundaries.

\subsection{Comparison of the quiet Sun velocity features with the radiance ones}

The fact that the feature sizes of the radiance images are larger than the velocity ones, needs more investigation. This result is in agreement with some previous works 


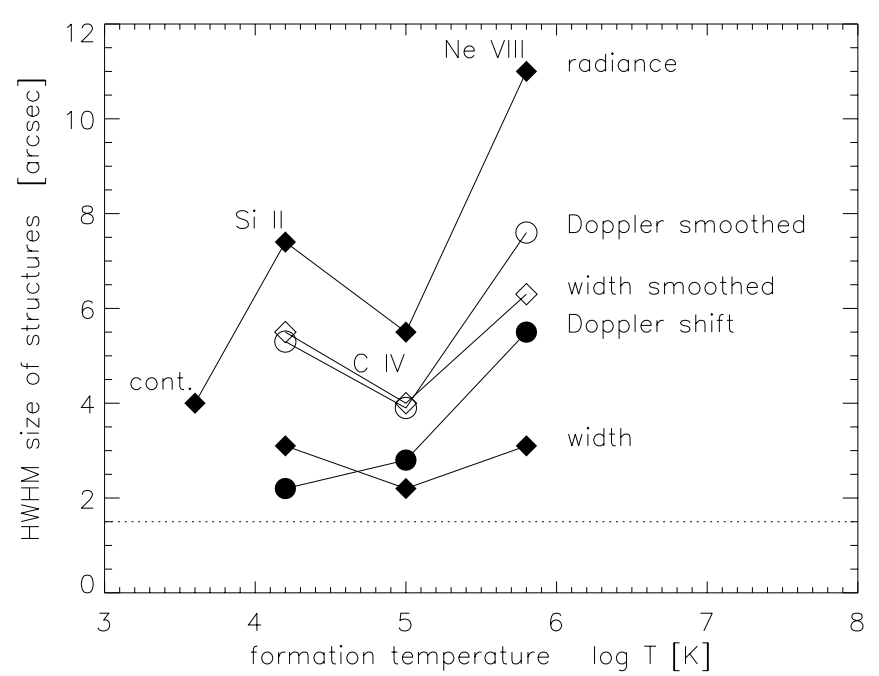

Fig. 7. $H W H M$ for the various ACFs of the SUMER data, as a function of formation temperature. The dotted line indicates the $H W H M$ for the SUMER images resolution limit (3").

(Gontikakis et al. 2001; Tsiropoula et al. 1994) for the transition region and the chromosphere. However, Dere et al. (1984) found that radiance structures along the slit of the HRTS-3 are smaller than the velocity ones in the C IV line. A possible explanation for this discrepancy may be due to the different lifetimes of radiance and velocity features. For the moment let us assume that the radiance features have a smaller scale than the velocity features. If now the time scale is smaller for the features seen in velocity than for those in radiance, the instrument might cover the full duration for a radiance structure across the slit during the raster process, but it might cover just a part of the corresponding velocity feature. The result will be a velocity feature diminished in the direction across the slit. However, the 2-D central part of the ACF is rather isotropic, showing that this stretching effect does not influence the shape of the features significantly.

The discrepancy between our result and the one of Dere et al. could be due to the fact that HRTS-3 and SUMER have different spatial and spectral resolution. The exposure time used by HRTS-3 was of $3 \mathrm{~s}$, much smaller than ours, where the intermittency of the transition region could modulate smaller radiance features.

However, this difference could be due to the smaller sample of data that was available to Dere et al. using HRTS data. To check this, we computed the $H W H M$ along and across the slit in the SUMER images using an 1-D ACF function. On the average, the radiance features were found to be larger than the velocity and non-thermal width ones, in both directions. In Fig. 8, we see that the $H W H M$ of the velocity along the slit, is larger than the one across it. In our (larger) sample we find that at $15 \%$ of the slit positions, the features in velocity are larger than in radiance. Thus, the Dere et al. result might be a selection effect, although more data are needed to investigate this further.

\subsection{Smallest structures in the low transition region}

The low values of the HWHM measured in the C IV line, coincide with stronger fluctuations. Due to these higher
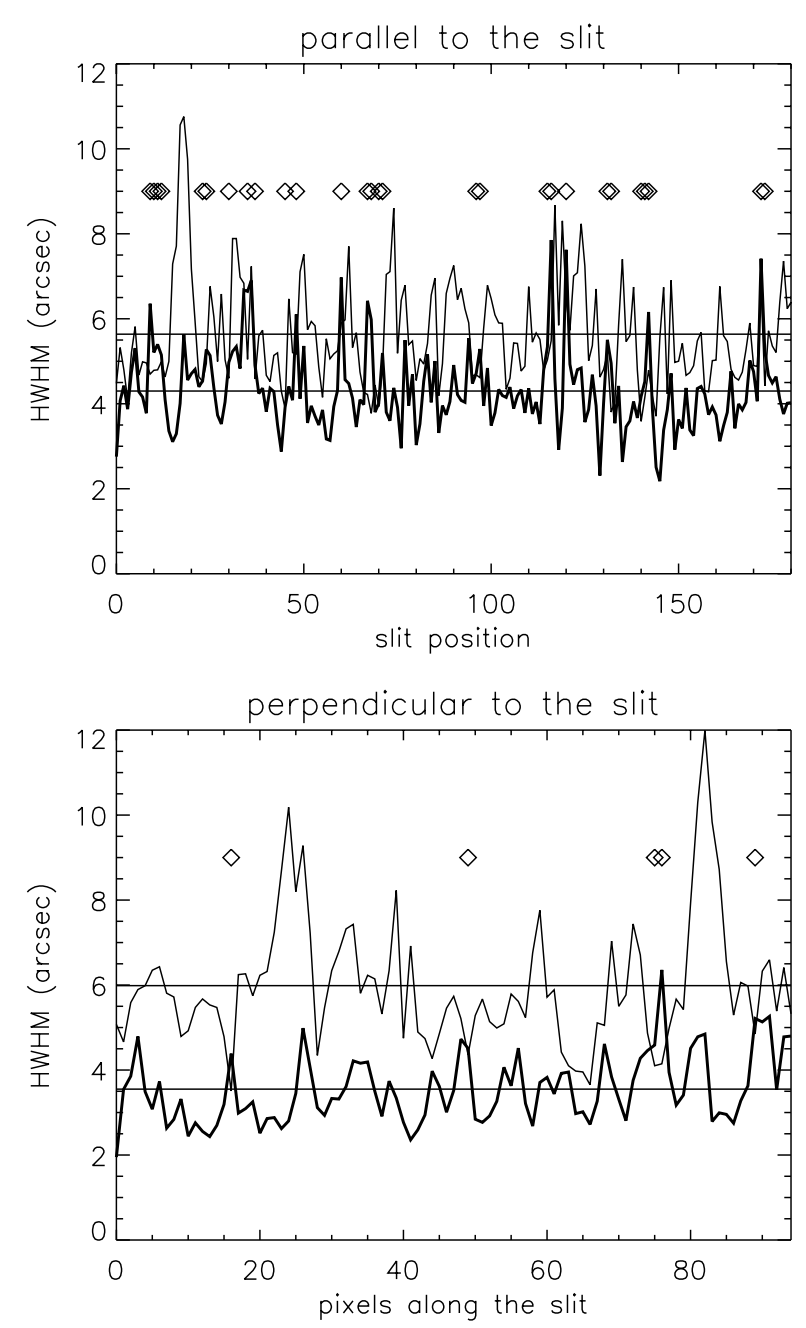

Fig. 8. Comparison of the 1-D ACF between radiance and dopplergrams of the $\mathrm{C}$ IV line (core component). In the first panel the $y$-axis is the $H W H M$ along the slit in seconds of arc, where the $x$-axis shows the slit position. Here also, the dopplergrams are smoothed to avoid the influence of the noise (see Sect. 3.2). The thin line represents the radiance $H W H M$, while the thick line shows the velocity $H W H M$. The two horizontal lines give the respective mean values. The diamonds indicate the positions where the radiance $H W H M$ is smaller than the velocity one.

radiance fluctuations in the network, the stucture may look more fragmented.

In order to quantify the radiance fluctuation, we divided the image into $3 \times 3$ square pixels. Then we calculated the relative variation of the radiance of these samples $\Delta f / f, f$ being the radiance in each square of $3 \times 3$ pixels. In Fig. 9, we show the histogram of $\Delta f / f$ for the radiance images of Si II, He II and C IV. The histogram shows that the relative variation of $\mathrm{Si} \mathrm{II} \mathrm{and} \mathrm{He}$ II is close to $5 \%$, whereas the variation for C IV is around $17 \%$. This indicates that the fluctuation of the radiance is more important at the level of C IV formation and produces a decrease of the HWHM in the same way noise does (see Sect. 3.2).

Similarly, Wilhelm et al. (1998) found that the spatial radiance variability was higher in the lower and middle transition region. In addition, the temporal variability was also found maximal in the middle transition region, (Brković et al. 2003), 

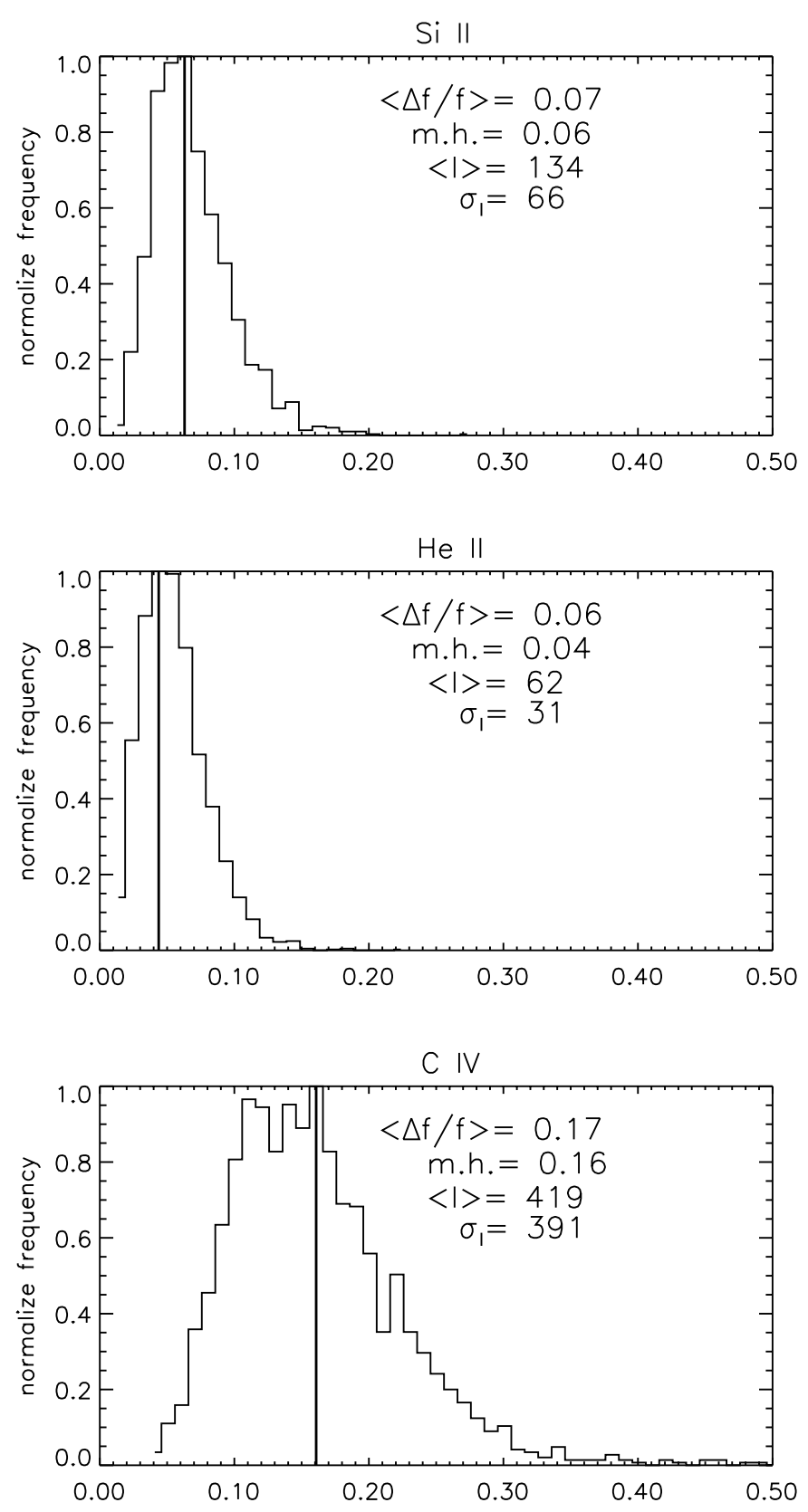

Fig. 9. Histogram of the relative variation of the $\Delta f / f$, for the Si II (left panel) and the C IV (right panel) lines. The C IV histogram has a more extended queue in the large values of $\Delta f / f$, indicating that the fluctuation of the intensities is more important in this image. In each panel, $\langle I\rangle$ is the mean radiance, $\sigma_{I}$ is the standard variation of it, $<\Delta f / f>$ is the mean of $\Delta f / f$. The maximum of the histogram is also indicated.

suggesting a close relation between the spatial and temporal variations.

\section{Analysis of the images of the tail component of the C IV line}

We repeated the analysis of the ACF for the images created by the tail component parameters of the Gaussian fits. In this case, the computation is influenced by two factors: first, the tail component parameters are more noisy, due to the relatively weak
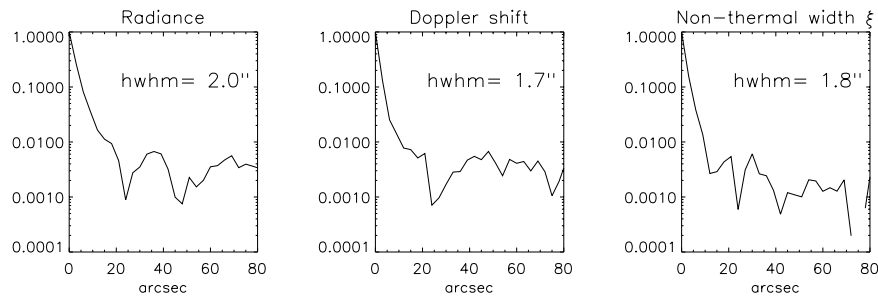

Fig. 10. ACF of the C IV images of the parameter of the tail spectral component. The first panel shows the ACF for the radiance images, the second of the dopplergrams and the third of the non-thermal width. The ACF curves of are produced by images in which we kept only the DGF pixels.

radiance of the tail component (only $1 / 3$ compared to the core; see Peter 2000, 2001); second, the tail component is basically restricted to the network (Peter 2000). Therefore the "image" of the tail component radiance, Doppler shift or width is patchy; the "image" is restricted to those regions, where a tail component is significantly detected.

The selection criteria applied are: (1) the $\chi_{\mathrm{r}}^{2}$ of the double Gaussian fit (DGF) should be less than 0.9 of the one of a single Gaussian fit (SGF). This means that the addition of a second Gaussian improves the fit. (2) The $\chi_{\mathrm{r}}^{2}$ must be smaller than 1.5, so that the fit is reliable (Peter 2000). The profiles where the two criteria meet, are called "two component regions", while the ones where only the second criterion is met are the "single component regions". The second Gaussian, for the cases where the second criterion is not satisfied (as for example in the inter-network), are so stretched that they reach the adjacent continuum (Peter 2000, Figs. 4a-c).

In Fig. 10 we see the shape of the ACF of the C IV images, where only the DGF points were included. The size of the features depends on the selection criteria. However, the values of the $H W H M$ are smaller than the core component ones and we found that the typical size of the tail component radiance features is smaller than the core component ones by a factor $\simeq 2$ to 2.5 .

The dopplergrams and the non-thermal width ACF $H W H M$ is of the same order as the one of the radiance image. However, the line profile parameters of line position and width have larger uncertainties, introduced by the fit, than the radiance (the position and width of a weak broad component below a strong narrow one is hard to determine). This is underlined by the fact that the $H W H M$ of $1.7^{\prime \prime}$ and $1.8^{\prime \prime}$ is very close to the resolution limit of 1.6" (see Sect. 3.2). Therefore, the feature sizes of the tail component radiance, position and width could look quite different with a better spatial resolution.

\section{Discussion}

\subsection{Size of radiance features as a function of temperature}

The $H W H M$ of the radiance features is generally increasing with the formation temperatures of the emission lines, which means with height, if one assumes a temperature stratification of the various transition region structures. This can be interpreted by the opening of the magnetic field lines when they 
reach higher levels of the atmosphere, as it is described in the Gabriel (1976) model. The increase of the size of bright features from the temperature minimum (continuum) to the upper chromosphere ( $\mathrm{Si}$ II line) continues in the low transition region, as we can see from the radiance images of the Si II and the C IV lines (Fig. 1). In the first image, the network elements are quite small and the image is dominated by the dark cells. However, in the C IV line, the dark cells are smaller and the network features are occupying a larger area.

However, the autocorrelation analysis revealed smaller structures in the case of the low transition region (in the C IV line) than in the upper chromosphere (the Si II line). The same behavior was found by Patsourakos et al. (1999), where the He I $584 \AA$ features, corresponding to chromospheric and lower transition region plasma, were larger than the ones of the low transition region in the O III $599 \AA$ and O V $630 \AA$ lines. The authors excluded the influence of the opacity of the He I line. This is in agreement with our results, since the Si II line is optically thin. Moreover, in contrast to the helium lines, the Si II line is not sensitive to coronal radiation, excluding an explanation based on coronal back radiation. It is very important to note that the ACF gives information about the structuring of the individual network elements and cannot estimate the real size of the network features.

In the transition region both the spatial and the temporal radiance variations are maximum (Fig. 9; Wilhelm et al. 1998; Brković et al. 2003; Patsourakos \& Vial 2002). The latter is due to the very short (down to seconds) time scales in the transition region. Short time scales also imply high spatial variability, if one assumes that structures next to each other are independent (this should be the case because of the very inefficient heat conduction across the magnetic field).

At higher temperatures, in the upper transition region and the lower corona, the time scales for cooling and conduction become much longer (up to hours). Therefore the increase of the feature size is picking up again and we see larger, streched out features.

We suggest that the minimum size of the structures in the transition region may be explained by the intermittence of the solar atmosphere which includes energy release at small temporal and spatial scales.

\subsection{Comparison of radiance features with the velocity ones}

The values of the $H W H M$ of the velocity features and the nonthermal widths are always smaller than the intensity ones.

As we described in Sect. 3.2, we further processed the dopplergrams, which are much more affected by the noise, even with the good statistics of the data. We are careful for the case of the Si II dopplergram, where, due to the very small values of the velocities, the relative uncertainties are important. Applying the autocorrelation method on a sample of the C IV image that includes only the bright network, we get the same result: the intensity features are larger than the velocity ones.

We can think of two possible explanations, one based on the fact that different structures may show different Doppler shifts, even when they have comparable intensities, and another based on the geometry of the loops.

In the first case, the two directions of flow (towards and away from the solar surface) expected in a siphon flow loop, are not present, or at least cannot be resolved in our low and middle transition region dopplergrams.

However, it is possible that one may detect many different flows in the dopplergrams, which may correspond to hardly distinguishable legs of loops in the radiance image. This may be so, because the loops could be heated by roughly the same amount of energy that is almost completely radiated away, resulting in similar loop radiances. Because of the geometry of the magnetic field, the heating may be asymmetric, inducing different flows in the different legs, which would lead to different Doppler shifts. This would correspond to packing different model loops as calculated, for example, by McClymont \& Craig (1987), Mariska (1988) or Hansteen (1993) together, with the different loops having comparable intensities but different net redshifts.

In the second case, we have to keep in mind that we observe just the flow along the line of sight. Since the flow follows the magnetic field geometry, the plasma flowing along nearly horizontal parts of the magnetic field will not be detectable, even if the region there is bright, while its flow will be better detected in the region where the magnetic field is vertical. For the case of the C IV line, if the network bright features are filled with small loops of length of the order of $\leq 10^{4} \mathrm{~km}$, as Dowdy et al. (1986) suggested, the redshift will be detectable only near the footpoints and not at the top. For small loops at $10^{5} \mathrm{~K}$, their scale height $(\simeq 2000 \mathrm{~km})$ is of the same order as their length and the intensity will be uniform along them. This explanation could fit our observations, if the loop length were larger than the resolution element, even if it couldn't be resolved, due to absence of contrast.

However, there is more to the interpretation of flows in loops than simply looking at the velocities. What we observe are the photons and, as shown in the loop models just mentioned above, one has to calculate the emission line spectra from the loop model to get the interpretation of the Doppler shifts right. There is a complex interaction between plasma flows, waves and density, which all contribute to the formation of the lines. Therefore more theoretical model work is needed for final conclusions.

It should be noticed that the smaller features of the dopplergrams, have already been detected in the upper chromosphere in C I (Dere et al. 1984) where they were interpreted as chromospheric jets.

\subsection{The non-thermal width}

We found that the features of large non-thermal width, $\xi$, are always smaller than the intensity features and similar to the velocity ones. What was said in the previous paragraph for the Doppler shift features can be also applied here, based on the calculations of Hansteen (1993), Hansteen et al. (1996) and Wikstøl et al. (1997). In these models, the redshift effect is produced by the propagation of compressive (MHD or purely 
acoustic) non-linear waves towards the surface of the Sun. The waves are generated by the energy release of nanoflares in the upper part of the loops. The effect of a compressive wave on a spectral profile depends on the angle between the line of sight and the direction of the wave (Eriksen \& Maltby 1967), which, in our case, is along the magnetic field. The fact that the Doppler shift and non-thermal width features are of the same order, could be due to the fact that they depend on the orientation of the magnetic field. We should note that a non-isotropic distribution of the line widths was excluded by previous authors (Chae et al. 1998), since the comparison of the line width at the disk centre and at the limb shows very small variation. However, in full disk scans of the C IV, we can see a very small, but measurable, variation of the line width of $\mathrm{C}$ IV as a function of $\cos \theta$ (Peter 1999, but see also Doyle et al. 2000, for a different result). Let us note that some turbulence regimes could also lead to non-isotropic distribution of un-resolved motions (e.g., Dmitruk et al. 2002; Gómez et al. 1992).

The interpretation of the spectral features of the tail component is impeded by the low signal-to-noise ratio. Since the ACF method is of statistical nature, part of the noise is smoothed out. However, the HWHM of the different spectral features is very close to the pixel size of the image and the interpretation cannot be straightforward for the case of the Doppler shifts and the line widths. The $H W H M$ of the radiance features of the tail component is much smaller than the one of the core component, at the same atmospheric level. A picture that could give an explanation, is that the funnels at the level of the low transition region $\left(\simeq 10^{5} \mathrm{~K}\right)$ are suppressed by the bunches of the small cool loops. The $H W H M$ of the tail component line width seems to be equivalent to the one of the core component. This indicates that the heating processes, involving turbulence formation, are similar. A possible explanation for this may be based on the interpretation of the tail components originating from coronal funnels, as suggested by Peter $(2000,2001)$. The feet of the (locally open) funnels may be squeezed by the surrounding small closed regions with strong magnetic fields. This could lead to a smaller size of the funnels at the level of the low transition region. Dere \& Mason (1993) suggested that the tail components are due to explosive events, which would also be compatible with our finding of smaller tail component features, since explosive events are small structures, mostly at or below the spatial resolution limit of current EUV spectrographs.

\section{Conclusions}

The size of the bright network features observed at different levels of the atmosphere, changes with height. This fits the picture of the magnetic field lines opening with height. However, there is an exception at the level of the middle transition region, due to the short time scales.

We found that the size of the features of the Doppler shift and Doppler width maps are always smaller than the corresponding radiance ones. Unresolved loops supporting plasma motions along them (either by siphon flows or acoustic waves) could be consistent with this image, since the strength of the observed Doppler shift (mostly redshift) should be small near the top where the magnetic field of the loop is horizontal.
Finally, the features in the images of the tail component of the C IV line were found to be much smaller than the ones of the core component. It may be that these features correspond to funnels crossing the Transition Region. Explosive events could also be of this scale. This fits the proposal of Peter (2000) that the tail and core components follow different physical processes.

Further work, with higher resolution observations, is needed to clarify the nature of these transition region features.

Acknowledgements. This program was partly financed by the Research Committee of the Academy of Athens and the Costopoulos Foundation. C.G. would like to thank the people at the Kiepenheuer Institut für Sonnenphysik for their warm hospitality. H.P. gratefully acknowledges the financial support of the Academy of Athens for a fruitful visit to Athens. We also thank Prof. C. E. Alissandrakis for his software support of this work and for his suggestions. SUMER is financially supported by DLR, CNES, NASA, and the ESA PRODEX programme. We thank our referee, Dr. K. Wilhelm, for his constructive comments, which improved the paper.

\section{References}

Andretta, V., Del Zanna, G., \& Jordan, S. D. 2003, A\&A, 400, 737

Arnaud, M., \& Rothenflug, R. 1985, A\&AS, 60, 425

Brault, J. W., \& White, O. R. 1971, A\&A, 13, 169

Brković, A., Peter, H., \& Solanki, S. K. 2003, A\&A, 403, 725

Brynildsen N., Brekke P., Fredvik T., et al. 1998, Sol. Phys., 181, 23

Chae, J., Schühle, U., \& Lemaire, P. 1998, ApJ, 505, 957

Charbonneau, P. 1995, ApJS, 101, 309

Dammasch, I. E., Wilhelm, K., Curdt, W., \& Hassler, D. M. 1999, A\&A, 346, 285

Dere, K. P., Bartoe, J.-D. F., \& Brueckner, G. E. 1984, ApJ, 281, 870

Dere, K. P., \& Mason, H. E. 1993, Sol. Phys., 217, 241

Domingo, V., Fleck, B., \& Poland, A. I. 1995, Sol. Phys., 162, 1

Doschek, G. A., Feldman, U., \& Bohlin, J. D. 1976, ApJ, 205, L117

Dowdy, J. F., Rabin, D., \& Moore, R. L. 1986, Sol. Phys., 105, 35

Doyle, J. G., Teriaca, L., \& Banerjee, D. 2000, A\&A, 356, 335

Dmitruk, P., Matthaeus, W. H., Milano, L. J., et al. 2002, ApJ, 575, 571

Eriksen, G., \& Maltby, P. 1967, ApJ, 148, 833

Feldman, U. 1983, ApJ, 275, 367

Feldman, U. 1987, ApJ, 320, 426

Feldman, U., Dammasch, I. E., \& Wilhelm, K. 2001, ApJ, 558, 423

Gabriel, A. H. 1976, Philos. Trans. R. Soc. London A, 281, 575

Gómez, D. O., \& Fontán, C. F. 1992, ApJ, 394, 662

Gontikakis, C., \& Dara, H. C. 2000, NewAR, 44, 599

Gontikakis, C., Dara, H. C., Alissandrakis, C. E., Zachariadis, Th. G., \& Vial, J.-C. 2001, A\&A, 378, 257

Handy, B. N., Acton, L. W., Kankellong, C. C., et al. 1999, Sol. Phys., 187, 229

Hansteen, V. H. 1993, ApJ, 402, 741

Hansteen, V. H., \& Leer, E. 1995, J. Geophys. Res., 100, 21577

Hansteen, V. H., Maltby, P., \& Malagoli, A. 1996, in Magnetic Reconnection in the Solar Atmosphere, ed. R. D. Bentley, \& J. T. Mariska, ASP Conf. Ser., 111, 116

Hassler, D. M., Dammasch, I. E., Lemaire, P., et al. 1999, Science, 283, 749

Judge P. G., \& McIntosh S. W. 1999, Sol. Phys., 190, 331 
Kjeldseth Moe O., \& Nicolas K. R. 1977, ApJ, 211, 579

Mariska, J. T. 1988, ApJ, 334, 489

Mariska, J. T. 1992, The Solar Transition Region (Cambridge: Cambridge University Press)

McClymont, A. N., \& Craig, I. J. D. 1987, ApJ, 312, 402

Patsourakos, S., Vial, J.-C., Gabriel, A. H., \& Bellamine, N. 1999, ApJ, 522, 540

Patsourakos, S., \& Vial J.-C. 2002, A\&A, 385, 1073

Peter, H., \& Judge, P. G. 1999, ApJ, 522, 1148

Peter, H. 1999, ApJ, 516, 490

Peter, H. 2000, A\&A, 360, 761
Peter, H. 2001, A\&A, 374, 1108

Reeves, E. M. 1976, Sol. Phys., 46, 53

Teriaca, L., Banerjee, D., \& Doyle, J. G. 1999, A\&A, 349, 636

Tsiropoula, G., Alissandrakis, C. E., \& Schmieder, B. 1994, A\&A, 285,294

Vernazza, J. E., Avrett, E. H., \& Loeser, R. 1981, ApJS, 45, 635

Wilhelm, K., Curdt, W., Marsch, E., et al. 1995, Sol. Phys., 162, 189

Wilhelm, K., Lemaire, P., Dammasch, I. E., et al. 1998, A\&A, 334, 685

Wikstøl, Ø., Judge, P. G., \& Hansteen, V. H. 1997, ApJ, 483, 972

Wikstøl, Ø., Judge, P. G., \& Hansteen, V. H. 1998, ApJ, 501, 895 